\title{
Surface exclusion and molecular mobility may explain Vroman effects in protein adsorption
}

Citation for published version (APA):

Willems, G. M., Hermens, W. T., \& Hemker, H. C. (1991). Surface exclusion and molecular mobility may explain Vroman effects in protein adsorption. Journal of Biomaterials Science-Polymer Edition, 2(3), 217226. https://doi.org/10.1080/09205063.1991.9756661

Document status and date:

Published: 01/01/1991

DOI:

10.1080/09205063.1991.9756661

Document Version:

Publisher's PDF, also known as Version of record

\section{Please check the document version of this publication:}

- A submitted manuscript is the version of the article upon submission and before peer-review. There can be important differences between the submitted version and the official published version of record.

People interested in the research are advised to contact the author for the final version of the publication, or visit the DOI to the publisher's website.

- The final author version and the galley proof are versions of the publication after peer review.

- The final published version features the final layout of the paper including the volume, issue and page numbers.

Link to publication

\footnotetext{
General rights rights.

- You may freely distribute the URL identifying the publication in the public portal. please follow below link for the End User Agreement:

www.umlib.nl/taverne-license

Take down policy

If you believe that this document breaches copyright please contact us at:

repository@maastrichtuniversity.nl

providing details and we will investigate your claim.
}

Copyright and moral rights for the publications made accessible in the public portal are retained by the authors and/or other copyright owners and it is a condition of accessing publications that users recognise and abide by the legal requirements associated with these

- Users may download and print one copy of any publication from the public portal for the purpose of private study or research.

- You may not further distribute the material or use it for any profit-making activity or commercial gain

If the publication is distributed under the terms of Article $25 \mathrm{fa}$ of the Dutch Copyright Act, indicated by the "Taverne" license above, 


\title{
Surface exclusion and molecular mobility may explain Vroman effects in protein adsorption
}

\author{
GEORGE M. WILLEMS*, WIM TH. HERMENS and \\ H. COENRAAD HEMKER \\ Cardiovascular Research Institute Maastricht, Biomedical Center, University of Limburg, P.O. Box \\ 616, 6200 MD Maastricht, The Netherlands
}

Received 4 June 1990; revised 19 September 1990

\begin{abstract}
Data on protein adsorption usually show that for increasing surface coverage the adsorption velocity decreases much faster than linearly. This contrasts to the classical Langmuir model with an adsorption velocity proportional to the number of unoccupied binding sites. It has been shown that this non-linearity may explain phenomena like transient adsorption of different proteins from a protein mixture or dilution-dependent changes in binding properties, collectively called Vroman effects. However, the molecular mechanisms explaining this non-linear behavior remain to be established.

A Monte Carlo simulation model is presented that incorporates steric hindrance, lateral mobility and mutual interactions of adsorbed molecules. Experimental data on the adsorption kinetics of prothrombin and annexin $\mathrm{V}$, a recently discovered anticoagulant protein, at phospholipid bilayers are analyzed with this model.

A major conclusion is that the steep decline in adsorption rates for increasing surface coverage can be explained, without assuming repulsive forces between adsorbed molecules, as a surface exclusion effect combined with lateral mobility of adsorbed molecules. The fact that annexin $\mathrm{V}$ shows this effect to a much lesser degree than prothrombin is tentatively explained by clustering of adsorbed annexin V molecules. A qualitative effect of lateral mobility on the adsorption characteristics, predicted by the model, is confirmed in experiments in which the fluidity of the bilayers was manipulated.
\end{abstract}

Key words: Vroman effect; protein adsorption; Monte Carlo simulation; ellipsometry.

\section{INTRODUCTION}

Although in the past 20 years an abundance of experimental data on the adsorption of proteins at liquid/solid interfaces has been reported, theoretical understanding of the mechanisms governing this process is still incomplete $[1,2]$. Observed adsorption kinetics are seldom in agreement with the classical principle of adsorption by mass action with independent binding sites. Instead, puzzling phenomena occur such as maximal adsorption of one of the components of a protein mixture for intermediate buffer concentrations or net desorption of protein after addition of a new component to a protein mixture. Because of Leo Vroman's pioneering work in this field [3, 4] and his more recent demonstration of consecutive adsorption and displacement of plasma proteins [5], these phenomena have been collectively called 'Vroman effects'.

Such transient adsorptions can still be understood from classical principles: from a mixture of an abundant component with low binding affinity and a scarce component with high binding affinity, the abundant component may first adsorb and then be gradually displaced by the scarce one [5]. However, it has repeatedly been

*To whom correspondence should be addressed. 
confirmed that dilution of a protein mixture may change the ratio of adsorbed quantities of different proteins [6-8] and this observation cannot be reconciled with classical binding theory. Recently it was shown that part of these effects can be qualitatively understood by assuming exponential relations between the secondorder sorption rate constants and surface coverage [9], but the mechanisms responsible for such relations remain to be specified. The purpose of this study is to present a model that explains such exponentially decreasing adsorption rates.

It has been demonstrated that by taking into account the space occupied by adsorbed protein molecules one may explain drastic effects on total surface coverage [10] or adsorption kinetics $[11,12]$. In the present study this concept is expanded by incorporation of surface diffusion and forces between adsorbed molecules. These aspects are integrated in a Monte Carlo simulation model with non-overlapping molecules. It is shown that this model may explain qualitative differences observed in the adsorption of prothrombin and annexin $\mathrm{V}$ to phospholipid bilayers.

\section{MATERIALS AND METHODS}

\section{Monte Carlo simulation of protein adsorption}

The adsorbing surface is represented by a square in the $x-y$ plane with edges of length $L$ (arbitrary units). In order to avoid boundary effects, periodic boundary conditions were used, i.e. the coordinates $(x+i \cdot L, y+j \cdot L)$ are identical for all integer values of $i$ and $j$. The adsorbing molecules are represented as discs with radius $R$ and their flat sides parallel to the adsorbing surface. The adsorption is simulated by random generation of coordinates and adsorption of the molecule at the given position if such adsorption does not result in overlap with already adsorbed molecules. This no-overlap condition does not necessarily refer to the adsorbed part of the protein. If, for instance, only a small part of the molecule contacts the surface it could refer to the protein bulk at some distance of the surface. It is a crucial aspect of the model and will cause increasingly more failures to adsorb for increasing surface coverage, also depending on the configuration of adsorbed molecules. This configuration is determined by the previous adsorptions, the surface mobility and the molecular interactions. Lateral diffusion of mutually interacting molecules is modelled as follows:

- The interaction between any two molecules is represented by a potential $V$ depending on the distance $d$ between the molecules.

A random steplength is generated and the potential $V$ is evaluated for 5 positions $s_{1} \cdots s_{5}$, i.e. the original position and one steplength in each of the four lateral directions: $V\left(s_{i}\right)=\sum_{j} V\left(s_{i}, d_{j}\right), i=1 \cdots s$, with the summation over all adsorbed molecules.

One of the steps $s_{1} \cdots s_{5}$ is chosen randomly with a probability proportional to $\operatorname{EXP}\left(-V\left(s_{i}\right) / k T\right)$ with $k$ the Boltzman constant and $T$ the temperature $[13,14]$.

As usual in Monte Carlo simulations, the statistical properties of the system parameters, for instance in our case the adsorption velocity, are obtained by repetitive sampling alternated with equilibration periods. Two situations are considered. First it is assumed that the mobility of adsorbed molecules is so high that, during adsorption, the configuration of adsorbed molecules remains in thermodynamic equilibrium. In this case the simulation proceeds by the following steps: 
For a given surface coverage the corresponding number of molecules $N_{\text {ads }}$ is placed on a regular hexagonal grid on the surface.

Then a large number $N_{\text {eq }}$ of diffusion steps is performed sequentially for each of the adsorbed molecules.

After this initial equilibration phase, the adsorption velocity is sampled repetitively by shooting a large number $N_{\text {try }}$ of molecules to the surface. The accessible area fraction $A$ is calculated as $A=$ (number of adsorptions) $/ N_{\text {try }}$. Adsorbed molecules are removed again in order to keep surface coverage constant.

Between subsequent samples of the adsorption velocity, the system traverses its phase space by performing $N_{\text {trav }}$ diffusion steps.

In the second case considered, the adsorbed molecules have too low mobility to maintain thermodynamic equilibrium. In that case the simulation proceeds as follows:

Total adsorption is divided in a large number of adsorption steps by specification of successive levels of surface coverage.

After each adsorption step a specified number of $N_{\text {dif }}$ diffusion steps is performed sequentially for each molecule and the adsorption velocity is determined from the fraction of successful adsorption steps.

The statistical properties of the system are determined by performing independent runs of the adsorption.

\section{Proteins and phospholipid bilayers}

Bovine prothrombin was prepared according to the method of Owen [15] and kept stored at $-20^{\circ} \mathrm{C}$ in $0.05 \mathrm{M}$ Tris- $\mathrm{HCl}$ buffer, $\mathrm{pH}=7.5$, containing $0.1 \mathrm{M} \mathrm{NaCl}$. Before the experiments, $3 \mathrm{mM} \mathrm{CaCl}_{2}$ was added to the buffer and prothrombin was incubated for $30 \mathrm{~min}$. This procedure ensured that the calcium-dependent molecular transition of prothrombin, required for binding to phospholipid membranes [16], was fully completed.

Annexin $V$, formerly called vascular anticoagulant protein (VAC $\alpha$ ) [17] was produced by recombinant DNA technique as described [18]. It was a kind gift of Dr. Ch. Reutelingsperger.

Dioleoylphosphatidylserine (DOPS) and dimyristoylphosphatidylserine (DMPS) were prepared by enzymatic conversion [19] from the corresponding phosphatidylcholines purchased from Sigma Chemical Co. At room temperature DOPS is in a liquid phase, allowing rapid lateral diffusion, whereas DMPS is in a solid state with restricted lateral mobility of adsorbed molecules.

\section{Ellipsometry}

Preparation of the phospholipid bilayers on reflecting silicon slides and ellipsometric measurements of the protein adsorptions at $20^{\circ} \mathrm{C}$ have been described in detail $[20,21]$. The adsorption experiments were performed in rapidly stirred Tris$\mathrm{HCl}$ buffer, $0.05 \mathrm{M}, \mathrm{pH}=7.5$, containing $0.1 \mathrm{M} \mathrm{NaCl}$ and $3 \mathrm{mM} \mathrm{CaCl}_{2}$.

\section{RESULTS}

The initial positions of the molecules placed on the surface differ considerably from the equilibrium distribution and the validity of the simulation will thus depend on a 
sufficiently high value of $N_{\text {eq }}$. For the cases presented it was verified that $N_{\text {eq }}=100,000$ was sufficiently high to ensure less than $10 \%$ difference between the parameter values obtained and the parameter values for $N_{\text {eq }}$ extrapolated to infinity. Another option that may affect the validity of the simulations is the magnitude of the total surface area in proportion to the molecular dimension. Small values of $L / R$ may considerably influence the obtained values of $V_{\text {ads }}$. Again it was verified that this effect can be neglected for values of $L / R>25$. In the simulations presented in this study, a surface with edges of 35 molecular diameters was chosen. Other values used were a diffusion step, chosen randomly with uniform probability between 0-0.03 molecular diameter, $N_{\text {try }}=100,000$ (25 times), $N_{\text {trav }}=39, N_{\text {dif }}=0$ (no mobility) or $N_{\text {dif }}=10$ (low mobility).

Figure 1 presents the attractive potential between molecules:

$$
\begin{aligned}
V / k T & =+\infty & & \text { for } d<0 \\
& =-5+d / d_{0} & & \text { for } 0<d<d_{0} ; d_{0}=0.03 R . \\
& =-4 /\left(d / d_{0}\right) & & \text { for } d_{0}<d<\infty .
\end{aligned}
$$

with $d$ the distance between the edges of the molecules, i.e. $d$ is the distance between the centers minus one molecular diameter.

This potential assumes infinitely strong repulsion for overlapping molecules (hard discs) and a short range attractive force between the edges of the molecules, proportional to $1 / d^{2}$, at larger distances. In order to avoid a singularity at $d=0$, it is assumed that for a thin shell $0<d<d_{0}$, with $d_{0}=0.03 R$, the attractive force is counteracted by a repulsive force, caused for instance by the compression of water molecules or a soft peripheral protein zone, such that the net force remains constant for $0<d<d_{0}$. Two cases are considered in the simulations. In the first case the attractive potential is taken zero (no interaction) and in the second case it is taken equal to the potential presented in Fig. 1 (strong attraction).

The adsorption velocity $V_{\text {ads }}$ is proportional to the freely accessible surface area $A$ and Fig. 2 shows schematically how $A$ is determined by surface coverage and

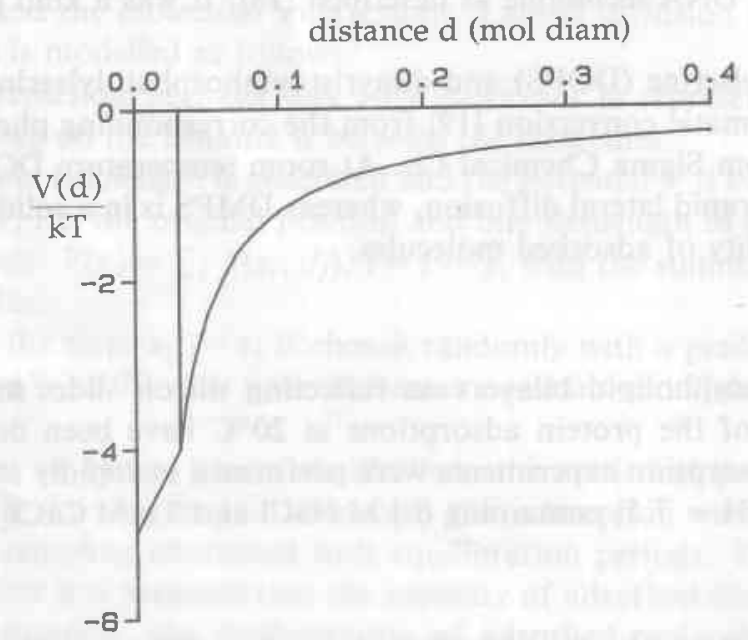

Figure 1. The attractive potential between molecules (see text). The distance $d$ between the edges of the discs is expressed in molecular diameters. 


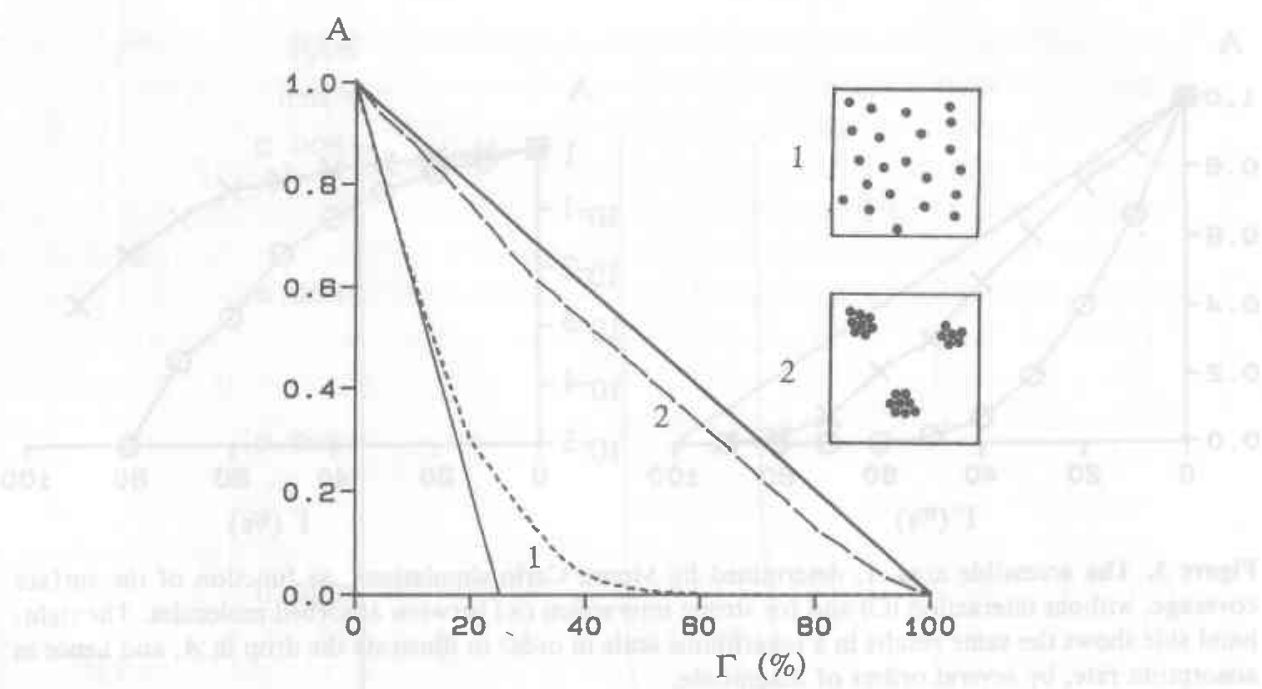

Figure 2. The influence of surface coverage and molecular configuration on the accessible area (schematically). The surface coverage $\Gamma$ is expressed as percentage of the maximal coverage of the surface.

molecular conformation. In a configuration of separately spaced molecules and a low surface coverage each molecule will exclude 4 times its own surface area. The accessible surface area will thus be roughly proportional to $A=1-4 \Gamma$. The excluded area's of different molecules will start to overlap for higher values of $\Gamma$ and a higher accessible area, indicated by the dotted line, will be obtained. In a configuration of closely packed clusters, however, the accessible surface will roughly change according to $A=1-\Gamma$.

Figure 3 shows the actual results of Monte Carlo simulations. Most important observation is that for increasing surface coverage the accessible surface area also decreases rapidly in the absence of molecular interactions. The right-hand side of Fig. 3 shows that the accessible area, and hence the adsorption velocity, drops over several orders of magnitude for increasing $\Gamma$. As the intrinsic adsorption rate indeed is mostly about two orders of magnitude higher than the transport-limited adsorption rate (cf. Fig. 5) this large decay is required before the intrinsic adsorption rate becomes observable. Also apparent is the shift of this decay to higher surface coverages for mutually attracting molecules caused by the clustering tendency.

Figure 4 presents the adsorption of prothrombin and annexin V to DOPS bilayers. The initial adsorption rates of both proteins are transport-limited, i.e. one does not observe the true intrinsic adsorption rates but the maximal transport rate of protein from the buffer to the surface that can be attained by the combined effects of diffusion and convection. For increasing surface coverage, however, the intrinsic adsorption rates rapidly decrease and drop below the transport limit and thereafter can actually be observed. Figure 4 shows that this occurs much earlier for prothrombin than for annexin $\mathrm{V}$.

This behavior is also shown in the simulation presented in Fig. 5. This figure shows so-called fractional adsorption rates, i.e. the adsorption rates normalized to the buffer concentrations: FAR $=V_{\mathrm{ads}} / C_{\mathrm{b}}$. Two cases are shown: A strong attracting potential and non-interacting molecules. The intrinsic FAR is proportional to 

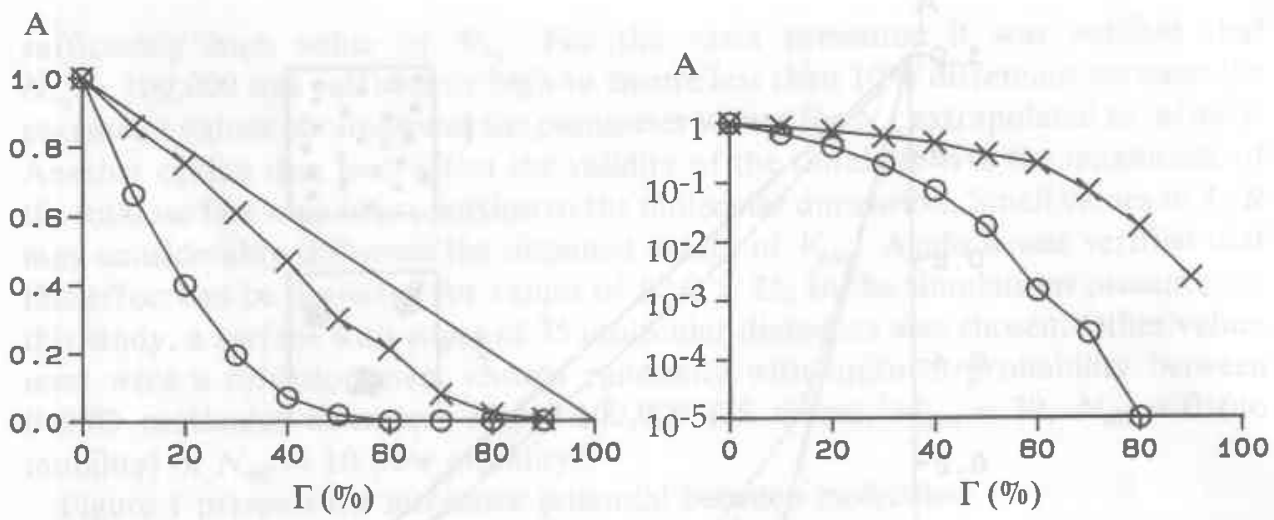

Figure 3. The accessible area $A$, determined by Monte Carlo simulations, as function of the surface coverage, without interaction $(O)$ and for strong interaction $(x)$ between adsorbed molecules. The righthand side shows the same results in a logarithmic scale in order to illustrate the drop in $A$, and hence in adsorption rate, by several orders of magnitude.
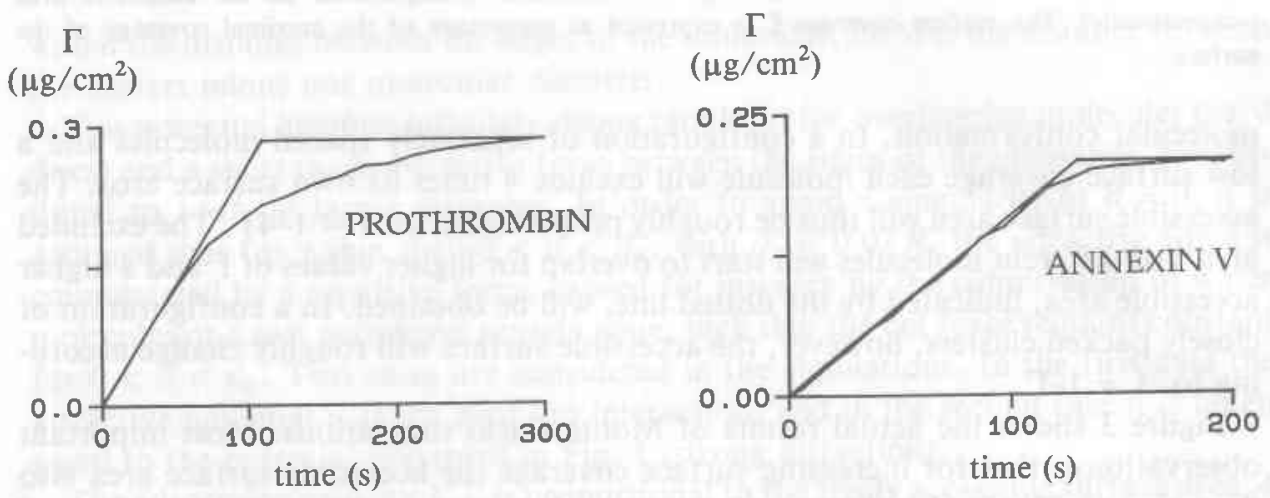

Figure 4. Adsorption kinetics of prothrombin and annexin V to DOPS bilayers. Buffer concentrations of both proteins were respectively 6 and $1 \mu \mathrm{g} / \mathrm{ml}$.

the accessible area: FAR $=k_{\mathrm{on}} \times A$. A value of $k_{\mathrm{on}}=0.1(\mathrm{~cm} / \mathrm{s})$ was used in this simulation. In agreement with Fig. 4 it is apparent that clustering (right curve) diminishes the effect of surface coverage on the adsorption rate. Generally, initial adsorption rates are transport rate limited and the adsorbed fractional rate FAR is related to the intrinsic rate $k_{\mathrm{on}} \times A$ and the transport rate limit $\mathrm{FAR}_{\mathrm{o}}$ by [20]:

$$
\mathrm{FAR}=\frac{\mathrm{FAR}_{\mathrm{o}}}{\mathrm{FAR}_{\mathrm{o}}+k_{\mathrm{on}} A} k_{\mathrm{on}} A .
$$

The solid curves in Fig. 5 present the dependence of the apparent observed FAR on surface coverage that would be found in the presence of the indicated transport limit. The relevant finding in Fig. 4 is reproduced: for increasing surface coverage the adsorption rate of one protein may drop below the transport limit considerably earlier than for another protein. Comparison of Figs 4 and 5 shows that annexin V molecules probably have a strong mutual attraction or clustering tendency.

Figure 6 shows Monte Carlo simulations on the effect of lateral mobility. The right-hand side shows that the lateral mobility has only a marginal effect on the 


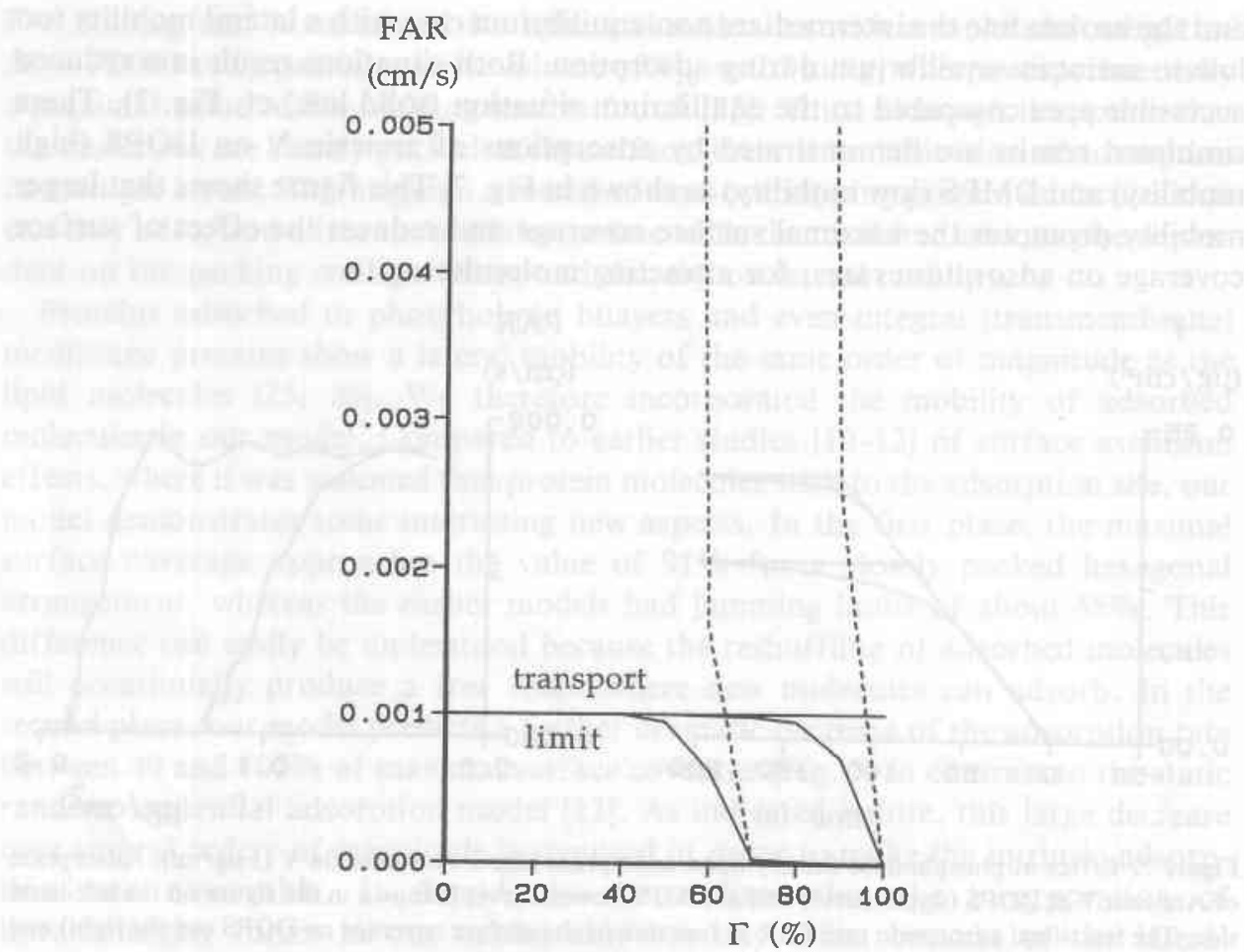

Figure 5. Illustration of the effect of the transport rate limit on apparent adsorption kinetics. Intrinsic fractional adsorption rates for strong attraction between adsorbed molecules (right curve) and without interaction (left curve) are indicated by dotted lines. The solid lines represent the apparent fractional rates, as would be observed in the presence of the indicated transport rate limit.

A

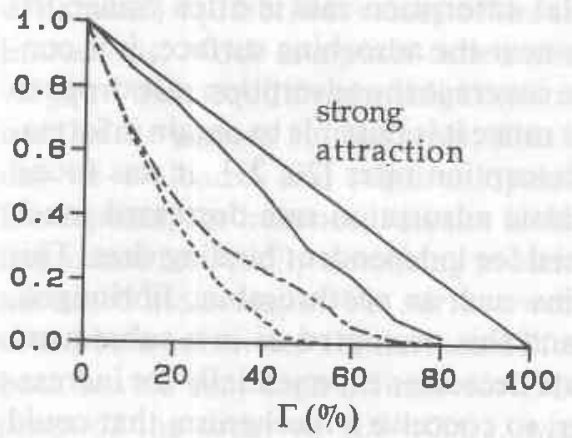

A

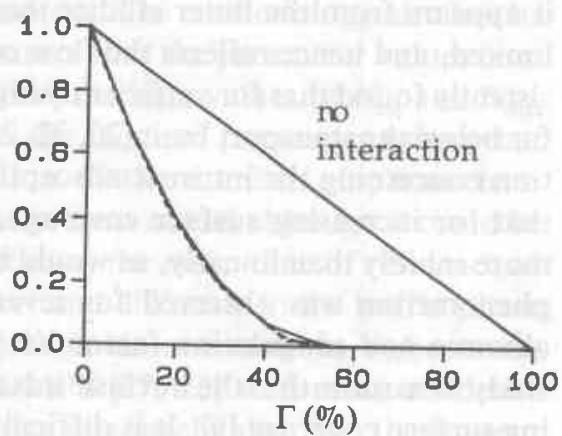

Figure 6. Simulations of the effect of limited mobility of adsorbed molecules on the accessible area. For non-interacting (right-hand side) and strongly attracting (left-hand side) molecules the accessible area without mobility $(\cdot \cdots)$, for low mobility $(\cdot \cdot \cdot \cdot)$ and for complete equilibration $(\square)$ is shown as function of the surface coverage.

accessible area if adsorbed molecules do not interact. In that case the molecules will maintain their random positions. For mutually attracting molecules, however, diffusion strongly promotes adsorption (left-hand figure) because the resulting more compact molecular configuration will increase the freely accessible area. The (lower) dotted line of Fig. 6 represents the situation without lateral mobility on the surface 
and the broken line the intermediate non-equilibrium case with a lateral mobility too low to maintain equilibrium during adsorption. Both situations result in a reduced accessible area compared to the equilibrium situation (solid line, cf. Fig. 3). These simulated results are demonstrated by adsorptions of annexin V on DOPS (high mobility) and DMPS (low mobility) as shown in Fig. 7. This figure shows that larger mobility promotes the maximal surface coverage and reduces the effect of surface coverage on adsorption rates, for attracting molecules.

$\Gamma$

$\left(\mu \mathrm{g} / \mathrm{cm}^{2}\right)$

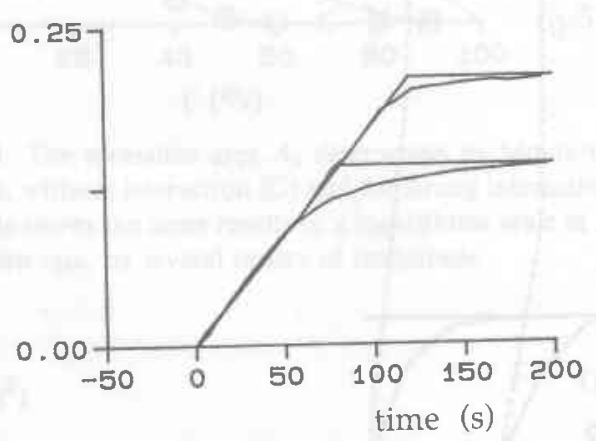

FAR

$(\mathrm{cm} / \mathrm{s})$

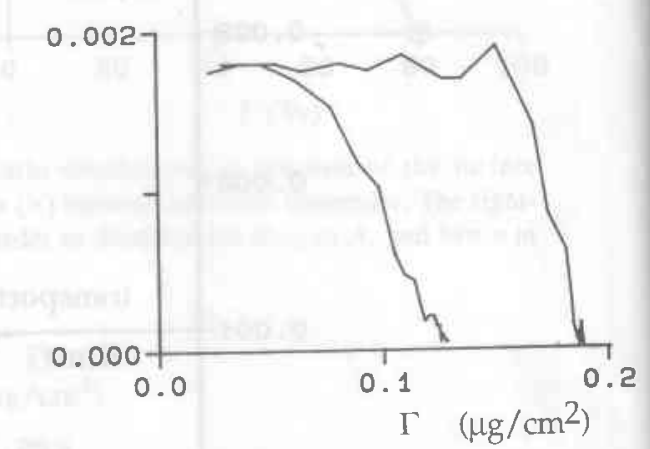

Figure 7. Effect of phospholipid fluidity upon adsorption kinetics of Annexin V $(1 \mu \mathrm{g} / \mathrm{ml})$. Adsorption of Annexin V at DOPS (upper curve) and at DMPS (lower curve) is shown in the figure on the left-hand side. The fractional adsorption rate FAR as function of the surface coverage on DOPS (on the right) and DMPS (on the left) is shown in the figure on the right-hand side.

\section{DISCUSSION}

Most studies on protein adsorption have focussed on the measurement of binding isotherms and relatively few have reported data on the kinetics of binding. Although it appears from the latter studies that the initial adsorption rate is often transportlimited, and hence reflects the flow conditions near the adsorbing surface, it is consistently found that for sufficiently high surface coverage the adsorption rate drops to far below the transport limit $[20,22,23]$. In this range it is possible to obtain information concerning the intrinsic adsorption and desorption rates $[20,21]$. It was found that for increasing surface coverage, the intrinsic adsorption rate decreased much more rapidly than linearly, as would be predicted for independent binding sites. This phenomenon was observed for several proteins such as prothrombin, fibrinogen, albumin and coagulation factor $\mathrm{Va}[20,21]$ and this prompted us in a subsequent study to assume that the intrinsic adsorption rate decreases exponentially for increasing surface coverage [9]. It is difficult however, to conceive a mechanism that could explain such inhibition of further adsorption by adsorbed protein molecules. Intuitively one feels that repulsive forces between molecules could cause such behavior. Electrostatic intermolecular forces however have an extremely short range in the order of the molecular diameter of proteins [24] in the conditions of high ionic strength used in these studies. This short range restricts the effectivity of this mechanism to situations with a high surface coverage, where steric hindrance alone already results in a steep decrease of the adsorption rate, as shown in this study.

We found for several proteins that maximal surface coverage is somewhat smaller than expected. For instance, for prothrombin a maximal surface coverage of 
$\Gamma=0.40 \mu \mathrm{g} / \mathrm{cm}^{2}$ is calculated for spherical molecules with a molecular weight of $M=72000$ and a partial specific volume of $V_{20}=0.7 \mathrm{ml} / \mathrm{g}$, whereas experimentally a maximal value of $\Gamma=0.35 \mu \mathrm{g} / \mathrm{cm}^{2}$ is found [25]. Thus, although it appears that the molecules are closely packed at the surface, there is no indication of adsorption in multiple layers. This suggests a model of non-overlapping protein molecules that can only adsorb on a free space of the surface while the total free area may be dependent on the packing configuration, and thereby on lateral mobility.

Proteins adsorbed to phospholipid bilayers and even integral (transmembrane) membrane proteins show a lateral mobility of the same order of magnitude as the lipid molecules $[25,26]$. We therefore incorporated the mobility of adsorbed molecules in our model. Compared to earlier studies [10-12] of surface exciusion effects, where it was assumed that protein molecules stick to the adsorption site, our model demonstrates some interesting new aspects. In the first place, the maximal surface coverage approaches the value of $91 \%$ for a closely packed hexagonal arrangement, whereas the earlier models had jamming limits of about $55 \%$. This difference can easily be understood because the reshuffling of adsorbed molecules will occasionally produce a free space where new molecules can adsorb. In the second place, our model predicts a further dramatic decrease of the adsorption rate between 40 and $100 \%$ of maximal surface coverage (Fig. 3) in contrast to the static random sequential adsorption model [12]. As indicated before, this large decrease over several orders of magnitude is required in order to make the intrinsic adsorption rate observable. It should also be realized that the incorporation of intramolecular forces in our model only has any effect because of the lateral mobility. The large changes shown in Figs. 6 and 7 are thus crucially dependent on this feature of our model.

The Monte Carlo simulation method has the advantage of simplicity and can also be applied to systems that do not allow analytical calculation of state parameters. In principle one samples the total phase space and may thus obtain true (thermodynamical) equilibrium values of the state parameters. The methodology also has its limitations. Much computer time is needed to ensure that the parameter values converge to their equilibrium values (see, for instance, the high values of $N_{\text {eq }}$ and $N_{\text {ads }}$ used in the present simulations). This also limits the range of parameter values that can be evaluated and requires optimal reduction of the number of parameters. Desorption, for instance, was not included in the present model although it could be important for readily desorbing proteins. In that case, the mean time of residence of adsorbed molecules on the surface would be short and diffusion in the buffer phase could be a substitute for surface diffusion. Prothrombin and annexin V, however, buth have a very high affinity for DOPS and DMPS $[27,28]$ with mean residence times of the order of $100-1000 \mathrm{~s}$, which excludes this mechanism for the present study. We also only considered attractive forces between the molecules in order to stress the fact that the rapid reduction of adsorption velocity for increasing surface coverage is not dependent on repulsive interactions. By counteracting any clustering tendency, however, such repulsive forces could have considerable effects. It should also be realized that the particles may assume dense packing but remain freely movable. They are not allowed to stick to each other. Such formation of fixed clusters of particles could well be induced by the orientational effects of the surface but this would only introduce marginal differences compared to the clustering allowed in the present study. 